\title{
On certain continued fractions involving two basic hypergeometric series
}

\author{
Dr. Jayprakash Yadav ${ }^{1}$, Vidhi Bhardwaj ${ }^{2}$ \\ ${ }^{I}$ Prahladrai Dalmia Lions College of Commerce and Economics, Mumbai, India. \\ ${ }^{2}$ D.S.J. College, Mumbai, India
}

Abstract: The object of this paper is to establish continued fraction for the ratio of two ${ }_{4} \phi_{3}$ series by the application of some known transformation formula in basic hyper geometric series.

Keywords and phrases: Basic hyper geometric series, Continued fractions and some known results in basic hyper geometric series.

The basic hyper geometric series is defined as

\section{Definitions And Notations}

${ }_{r} \phi_{s}\left[\begin{array}{l}(a) ; z \\ (b)\end{array}\right]=\sum_{n=0}^{\infty} \frac{((a) ; q)_{n} z^{n}}{((b) ; q)_{n}(q ; q)_{n}}$,

where for the convergence of the series, we have $0<|q|<1$ and $|z|<1$ if $\mathrm{r}=\mathrm{s}+1$, and for any $\mathrm{z}$ if $r \leq s$.

$((a) ; q)_{n}=\left(a_{1} ; q\right)_{n}\left(a_{2} ; q\right)_{n} \ldots\left(a_{r} ; q\right)_{n}$,

$((b) ; q)_{n}=\left(b_{1} ; q\right)_{n}\left(b_{2} ; q\right)_{n} \ldots\left(b_{s} ; q\right)_{n}$.

For real or complex, a, $q<1$, the $q$-shifted factorial is defined by

$(a, q)_{n}=\left\{\begin{array}{lll}1 & \text {; if } & n=0 \\ (1-q)(1-a q) \ldots\left(1-a q^{n-1}\right) & \text {; if } & n \in N .\end{array}\right.$

We will also use the following results in our analysis:

${ }_{4} \phi_{3}\left[\begin{array}{l}q^{-n}, a, b, c ; q \\ d, e, f\end{array}\right]=\frac{(e / a, f / a)_{n}}{(e, f)_{n}} a^{n}{ }_{4} \phi_{3}\left[\begin{array}{l}q^{-n}, a, d / b, d / c ; q \\ d, a q^{1-n} / e, a q^{1-n} / f\end{array}\right]$

and

${ }_{8} \phi_{7}\left[\begin{array}{c}\mu, q \mu^{\frac{1}{2}},-q \mu^{\frac{1}{2}}, a, b, c, d q^{n}, e q^{n} ; \frac{d e}{a b c} \\ \mu^{\frac{1}{2}},-\mu^{\frac{1}{2}}, \mu q / a, \mu q / b, \mu q / c, e, d\end{array}\right]=\frac{(a q / f, b q / f, c q / f, a b c / f ; q)_{\infty}}{(a b q / f, a c q / f, b c q / f, q / f ; q)_{\infty}} \phi_{3}\left[\begin{array}{l}q^{-n}, a, b, c ; q \\ d, e, f\end{array}\right]$

where $\mu=a b c / f, d e f=a b c q^{1-n}$ [ Gasper and Rahman [2]; Appiii(iii.20) ] 


\section{Main Results}

$(a)_{4} \phi_{3}\left[\begin{array}{l}q^{-n}, a, b, c: q \\ d, e, f\end{array}\right] /{ }_{4} \phi_{3}\left[\begin{array}{l}q^{-n}, a q, b, c: q \\ d q, e, f\end{array}\right]=1+\frac{\alpha_{0}}{\beta_{0}-1+} \frac{\gamma_{0}}{\beta_{1}-1+\beta_{2}-1+} \frac{\alpha_{1}}{1+} \frac{\alpha_{2}}{\beta_{2}} \frac{\gamma_{2}}{1+\ldots .}$

Where $\alpha_{i}=\frac{d q^{2 i+1}\left(1-q^{i-n}\right)\left(1-b q^{i}\right)\left(1-c q^{i}\right)}{\left(1-d q^{2 i}\right)\left(1-d q^{2 i+1}\right)\left(1-e q^{i}\right)\left(1-f q^{i}\right)}, \beta_{i}=\frac{a^{2} q^{2}}{(a q-e)(a q-f)\left(1-e q^{i}\right)\left(1-f q^{i}\right)}$

And $\gamma_{i}=\frac{e f}{b c} \frac{\left(1-d q^{i+n+1}\right)\left(1-a q^{i+1}\right)\left(b-d q^{i+1}\right)\left(c-d q^{i+1}\right) q^{i-2 n+1}}{\left(1-d q^{2 i+1}\right)\left(1-d q^{2 i+1}\right)\left(e-a q^{2-n+i}\right)\left(f-a q^{2-n+i}\right)\left(1-e q^{i}\right)\left(1-f q^{i}\right)}$.

(b)

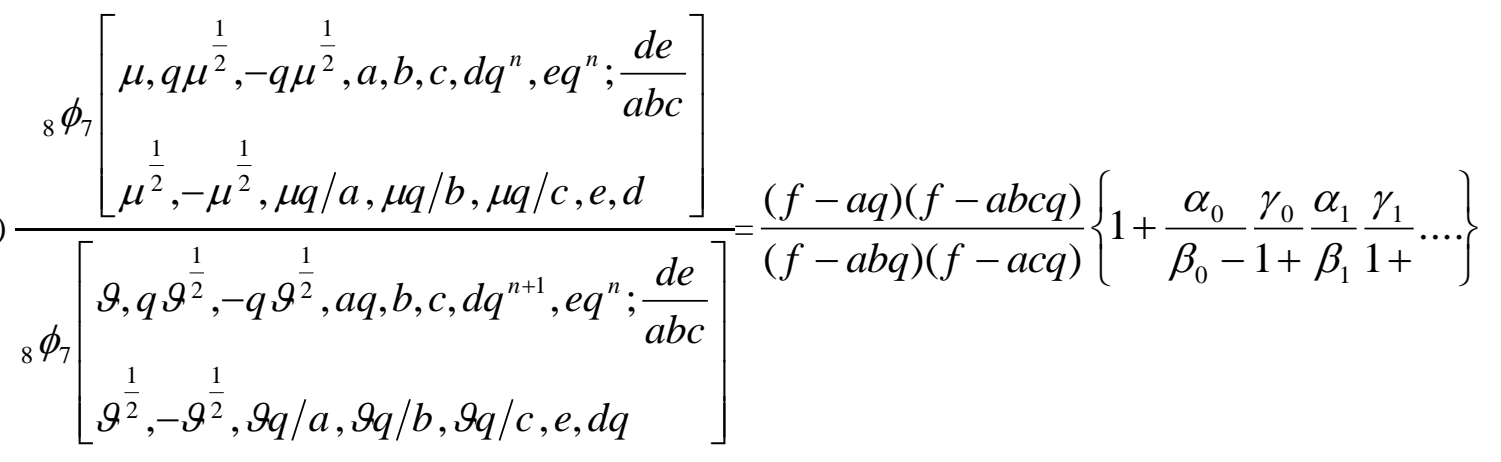

(7)

$\mu=a b c / f, \operatorname{def}=a b c q^{1-n}, \vartheta=a b c q / f$, def $=a b c q^{1-n}$.

Where $\alpha_{i}=\frac{d q^{2 i+1}\left(1-q^{i-n}\right)\left(1-b q^{i}\right)\left(1-c q^{i}\right)}{\left(1-d q^{2 i}\right)\left(1-d q^{2 i+1}\right)\left(1-e q^{i}\right)\left(1-f q^{i}\right)}, \beta_{i}=\frac{a^{2} q^{2}}{(a q-e)(a q-f)\left(1-e q^{i}\right)\left(1-f q^{i}\right)}$

and $\quad \gamma_{i}=\frac{\left(1-d q^{i+n+1}\right)\left(1-a q^{i+1}\right)\left(b-d q^{i+1}\right)\left(c-d q^{i+1}\right)}{\left(1-d q^{2 i+1}\right)\left(1-d q^{2 i+2}\right)\left(1-e q^{i}\right)\left(1-f q^{i}\right)\left(e-a q^{i-n+i}\right)\left(f-a q^{2-n+i}\right) b c}$

Proof of (a): $\quad$ For $i \geq o$,

Let $H_{i}={ }_{4} \phi_{3}\left[\begin{array}{l}q^{-n+i}, a q^{i}, b q^{i}, c q^{i}: q \\ d q^{2 i}, e q^{i}, f q^{i}\end{array}\right] \quad$ and $\quad F_{i}={ }_{4} \phi_{3}\left[\begin{array}{l}q^{-n+i}, a q^{i+1}, b q^{i}, c q^{i}: q \\ d q^{2 i+1}, e q^{i}, f q^{i}\end{array}\right]$

So,

$H_{i}-F_{i}=\frac{d q^{2 i+1}\left(1-q^{i-n}\right)\left(1-b q^{i}\right)\left(1-c q^{i}\right)}{\left(1-d q^{2 i}\right)\left(1-d q^{2 i+1}\right)\left(1-e q^{i}\right)\left(1-f q^{i}\right)} H_{i+1}$

This gives $\frac{H_{i}}{F_{i}}=1+\frac{\alpha_{i}}{F_{i} / H_{i+1}}$,

Where $\alpha_{i}=\frac{d q^{2 i+1}\left(1-q^{i-n}\right)\left(1-b q^{i}\right)\left(1-c q^{i}\right)}{\left(1-d q^{2 i}\right)\left(1-d q^{2 i+1}\right)\left(1-e q^{i}\right)\left(1-f q^{i}\right)}$.

Let us transform $F_{i}$ and $H_{i}$ by means of the transformation formula (4) we get: 


$$
\begin{aligned}
F_{i}= & \frac{(e / a q)_{n}(f / a q)_{n}}{\left(e q^{i}\right)_{n}\left(f q^{i}\right)_{n}}\left(a q^{i+1}\right)^{n}{ }_{4} \phi_{3}\left[\begin{array}{l}
q^{i-n}, a q^{i+1}, d q^{i+1} / b, d q^{i+1} / c ; q \\
d q^{2 i+1}, a q^{2-n+1} / e, a q^{2-n+} / f
\end{array}\right], \\
H_{i}= & \frac{(e / a q)_{n}(f / a)_{n}}{\left(e q^{i}\right)_{n}\left(f q^{i}\right)_{n}}\left(a q^{i}\right)^{n}{ }_{4} \phi_{3}\left[\begin{array}{l}
q^{i-n}, a q^{i}, d q^{i} / b, d q^{i} / c ; q \\
d q^{2 i}, a q^{1-n+i} / e, a q^{1-n+i} / f
\end{array}\right], \\
H_{i+1}= & \frac{(e / a q)_{n}(f / a)_{n}}{\left(e q^{i+1}\right)_{n}\left(f q^{i+1}\right)_{n}}\left(a q^{i+1}\right)^{n}{ }_{4} \phi_{3}\left[\begin{array}{l}
q^{i-n+1}, a q^{i+1}, d q^{i+1} / b, d q^{i+1} / c ; q \\
d q^{2 i+2}, a q^{2-n+i} / e, a q^{2-n+i} / f
\end{array}\right]
\end{aligned}
$$

So,

$$
\begin{aligned}
& \frac{H_{i+1}}{(1-e / a q)(1-f / a q)}-\left(e q^{i}\right)\left(f q^{i}\right) F_{i} \\
& =\frac{\left(1-a q^{i+1}\right)\left(1-d q^{i+1} / b\right)\left(1-d q^{i+1} / c\right)(e / a q)_{n}(f / a q)_{n}\left(a q^{i+2}\right)^{n}\left(1-d q^{i+n+1}\right) q^{i+1}}{q^{2 n}\left(1-d q^{2 i+1}\right)\left(1-d q^{2 i+2}\right)\left(1-a q^{2-n+i} / e\right)\left(1-a q^{2-n+i} / f\right)\left(e q^{i+1}\right)_{n}\left(f q^{i+1}\right)_{n}\left(1-d q^{2 i+1}\right)} \times \\
& \sum_{r=0}^{\infty} \frac{\left(q^{i-n+1}\right)_{r-1}\left(a q^{i+1}, d q^{i+1} / b, d q^{i+1} / c\right)_{r} q^{r}}{\left(d q^{2 i+2}\right)_{r}\left(a q^{2-n+i} / e, a q^{2-n+i} / f\right)_{r}},
\end{aligned}
$$

This gives the following equation:

$$
\begin{aligned}
& \frac{F_{i}}{H_{i+1}}=\frac{1}{(1-e / a q)(1-f / a q)\left(1-e q^{i}\right)\left(1-f q^{i}\right)}- \\
& \frac{\left(1-a q^{i+1}\right)\left(1-d q^{i+1} / b\right)\left(1-d q^{i+1} / c\right)\left(1-d q^{i+n+1}\right) q^{i+1-2 n}}{\left(1-d q^{2 i+1}\right)\left(1-d q^{2 i+2}\right)\left(1-a q^{2-n+i} / e\right)\left(1-a q^{2-n+i} / f\right)\left(e q^{i}\right)_{n}\left(f q^{i}\right)_{n}} \frac{F_{i+1}}{H_{i+1}},
\end{aligned}
$$

This can be written as $\frac{F_{i}}{H_{i+1}}=\beta_{i}-\frac{\gamma_{i}}{H_{i+1} / F_{i+1}}$, where $\beta_{i}$ and $\gamma_{i}$ are mentions above.

Now using (1) and (2) repeatedly, for $i \geq 0$ we get (6).

\section{Proof of $(\mathbf{b})$ :}

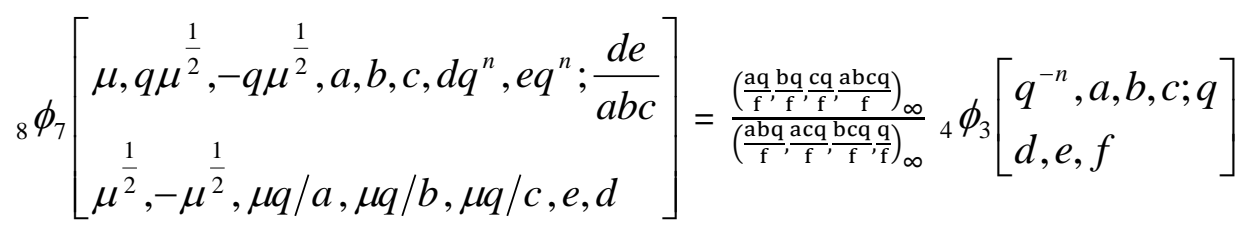

Where $\mu=\frac{a b c}{f}$, def $=a b c q^{1-n}$.

Let us replace $a$ by aq and $d$ by dq in the above equation, we get the following: 


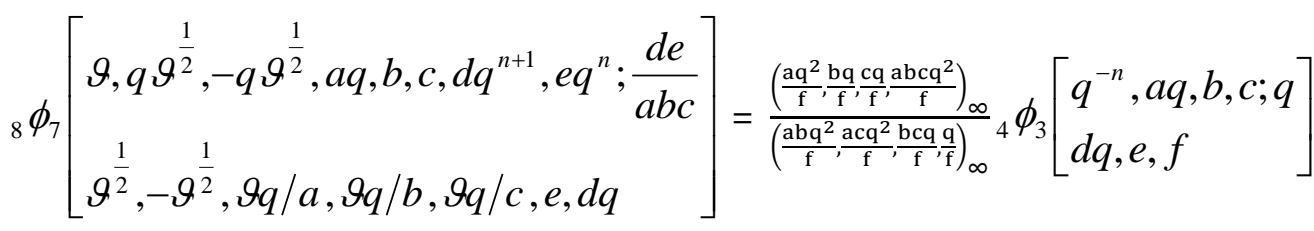

Now dividing equation (15) by equation (16) we get the following equation:

$$
\begin{aligned}
& \frac{{ }_{8} \phi_{7}\left[\begin{array}{l}
\mu, q \mu^{\frac{1}{2}},-q \mu^{\frac{1}{2}}, a, b, c, d q^{n}, e q^{n} ; \frac{d e}{a b c} \\
\mu^{\frac{1}{2}},-\mu^{\frac{1}{2}}, \mu q / a, \mu q / b, \mu q / c, e, d
\end{array}\right]}{{ }_{8} \phi_{7}\left[\vartheta, q \vartheta^{\frac{1}{2}},-q \vartheta^{\frac{1}{2}}, a q, b, c, d q^{n+1}, e q^{n} ; \frac{d e}{a b c}\right]}=\frac{(f-a q)(f-a b c q)}{(f-a b q)(f-a c q)} \frac{{ }_{4} \phi_{3}\left[\begin{array}{l}
q^{-n}, a, b, c ; q \\
d, e, f
\end{array}\right]}{{ }_{4} \phi_{3}\left[\begin{array}{l}
q^{-n}, a q, b, c ; q \\
d q, e, f
\end{array}\right]} \\
& {\left[\vartheta^{\frac{1}{2}},-\vartheta^{\frac{1}{2}}, \vartheta q / a, \vartheta q / b, \vartheta q / c, e, d q\right]}
\end{aligned}
$$

Now using result (6) on the right hand side we get the result (7).

\section{References}

[1]. Andrews, G.E., Askey R. and Roy, Ranjan. Special Functions, Cambridge University Press, Cambridge, 1999.

[2]. Agarwal, R.P. (1996): Resonance of Ramanujan's Mathematics, vol.II, New Age International (P) Limited, New Delhi.

[3]. A.M. Mathai and R.K. Saxena, Generalized Hypergeometric Functions with Applications in Statistics and Physical Sciences. Springer-Verlag, Berlin (1973)

[4]. Andrews, G.E. and Berndt, B.C. (2005): Ramanujan's Lost Notebook,Part1,Springer, New York

[5]. Gasper, G. and Rahman, M. (1990): Basic hyper geometric series, Cambridge University Press, Cambridge.

[6]. Karlsson, P.W. Hypergeometric functions with integral parameter difference, J. Math. Phys. 12 (1971), $270-271$.

[7]. Slater, L.J. (1966): Generalized hyper geometric functions, Cambridge University Press, Cambridge. 